\title{
Addiction and Smoking Cessation
}

\author{
Hossain $\mathrm{MD}^{1}$, Islam SAHMM ${ }^{2}$, Rashid $\mathrm{MM}^{3}$, Chowdhury MAI ${ }^{4}$, Bennoor $\mathrm{KS}^{5}$, Patwary $\mathrm{MI}^{6}$
}

\begin{abstract}
Cigarette smoke contains a deadly mix of more than 7,000 chemicals, hundreds are toxic and about 70 can cause cancer. Cigarette smoke can cause serious health problems, numerous diseases and death. Fortunately, people who stop smoking greatly reduce their risk for disease and premature death. Although the health benefits are greater for people who stop at earlier ages, cessation is beneficial at all ages. There are various methods and approaches in quitting smoking. Currently, there are about 1.3 billion smokers the world, most (84\%) of them in developing countries.If current smoking trends continue, tobacco will kill 10 million people each year by 2020 .
\end{abstract}

\section{Introduction}

Tobacco contains the chemical nicotine. Smoking cigarettes can lead to nicotine addiction. The addiction begins when nicotine acts on nicotinic acetylcholine receptors to release neurotransmitters such as dopamine, glutamate and gamma-aminobutyric acid. Cessation of smoking leads to symptoms of nicotine withdrawal such as

anxiety and irritability. Professional smoking cessation support methods generally endeavour to address both nicotine addiction and nicotine withdrawal symptoms ${ }^{1,2}$.

1. Corresponding Author: Md. Delwar Hossain Assistant Professor, Respiratory Medicine Sylhet MAG Osmani Medical College, Sylhet.

2. SAHM Mesbahul Islam Assistant Professor, Respiratory Medicine Sylhet MAG Osmani Medical College, Sylhet.

3. Md. Mamunur Rashid Indoor Medical officer National Institute of Diseases of the Chest and Hospital Mohakhali, Dhaka.

4. Md. Ashfaqul Islam Chowdhury Assistant Professor, Medicine Sylhet MAG Osmani Medical College, Sylhet

5. Kazi Saifuddin Bennoor Assistant Professor, Respiratory Medicine National Institute of Diseases of the Chest and Hospital, Mohakhali

6. Md. Ismail Patwary

Professor and Head, Department of Medicine Sylhet MAG Osmani Medical College, Sylhet
It takes between 6 to 12 weeks post quitting before the amount of nicotinic receptors in the brain return to the level of a non-smoker ${ }^{1,3}$.

\section{Nicotine Dependence}

- Nicotine is the psychoactive drug in tobacco products that produces dependence. Most smokers are dependent on nicotine.

- Nicotine dependence is the most common form of chemical dependence in the world.

- Quitting smoking is difficult and may require multiple attempts. Users often relapse because of stress, weight gain and withdrawal symptoms.

- Nicotine withdrawal symptoms include irritability, anxiety, difficulty concentrating and increased appetite.

Health Benefits of Cessation

Smoking cessation is associated with the following health benefits $^{4,5}$ :

- Smoking cessation lowers the risk for lung and other types of cancer.

- Smoking cessation reduces the risk for coronary heart disease, stroke and peripheral vascular disease. Coronary heart disease risk is substantially reduced within 1 to 2 years of cessation.

- Smoking cessation reduces respiratory symptoms, such as coughing, wheezing, and shortness of breath. The rate of decline in lung function is slower among persons who quit smoking.

- Smoking cessation reduces the risk of developing chronic obstructive pulmonary disease (COPD), one of the leading causes of death in the world.

- Smoking cessation by women during their reproductive years reduces the risk for infertility. Women who stop smoking during pregnancy also reduce their risk of having a low birth weight baby.

\section{Symptoms of smoking}

Nicotine use can have many different effects on body functions, both positive and negative. Nicotine acts as both a stimulant and depressant on the body ${ }^{6}$. The use of nicotine:

- Decreases the appetite

- Boosts mood and may even relieve minor depression. 
- Raises the blood level of blood sugar and increases insulin production.

- Increases bowel activity, saliva, and phlegm.

- Increases heart rate by around 10 to 20 beats per minute.

- Increases blood pressure by 5 to $10 \mathrm{mmHg}$.

- May cause sweating, nausea, and diarrhea.

- Stimulates memory and alertness.

Symptoms of smoking withdrawal

Symptoms of nicotine withdrawal generally start within 2 3 hours after the last tobacco use and will peak about 2 - 3 days later. Symptoms may be severe, depending on how long you smoked and how many cigarettes you smoked each day $^{2,7}$. Common symptoms include:

Symptoms and duration of nicotine withdrawal

\begin{tabular}{ll}
\hline Craving for tobacco & 3 to 8 weeks \\
Dizziness & Few days \\
Insomnia & 1 to 2 weeks \\
Headaches & 1 to 2 weeks \\
Chest discomfort & 1 to 2 weeks \\
Constipation & 1 to 2 weeks \\
Irritability & 2 to 4 weeks \\
Fatigue & 2 to 4 weeks \\
Cough or nasal drip & Few weeks \\
Lack of concentration & Few weeks \\
Hunger & Up to several weeks \\
Depression & Up to 3 years \\
\hline
\end{tabular}

A milder form of nicotine withdrawal that involves some or all of these symptoms can occur when a smoker switches from regular to low-nicotine cigarettes or significantly cuts down on the number of cigarettes smoked ${ }^{8}$.

Symptoms of nicotine withdrawal can mimic, disguise, or worsen the symptoms of other psychiatric problems.

\section{Methods to Quit Smoking}

Unassisted methods

As it is common for ex-smokers to have made a number of attempts to stop smoking before achieving long-term abstinence, identifying which approach or technique is eventually most successful is difficult, it has been estimated, for example, that "only about $4 \%$ to $7 \%$ of people are able to quit smoking on any given attempt without medicines or other help.". However, in analysing

a 1986 U.S. survey, Fiore et al. (1990) found that 95\% of former smokers who had been abstinent for 1-10 years had made an unassisted last quit attempt. The most frequent unassisted methods were "cold turkey" and "gradually decreased number" of cigarettes. A 1995 meta-analysis estimated that the quit rate from unaided methods was $7.3 \%$ after an average of 10 months of follow-up ${ }^{6,9}$.

\section{Cold turkey}

"Cold turkey" is a colloquial term indicating abrupt withdrawal from an addictive drug, and in this context indicates sudden and complete cessation of all nicotine use. In three studies, it was the quitting method cited by $76 \%, 85 \%$, or $88 \%$ of long-term successful quitters. In a large British study of ex-smokers in the 1980s, before the advent of pharmacotherapy, $53 \%$ of the ex-smokers said that it was "not at all difficult" to stop, $27 \%$ said it was "fairly difficult" and the remaining 20\% found it very difficult ${ }^{10}$.

\section{Healthcare provider and system interventions}

Interventions delivered via healthcare providers and healthcare systems have been shown to improve smoking cessation among people who visit those providers ${ }^{11}$.

In one systematic review and meta-analysis, multi-component interventions increased quit rates in primary care settings. "Multi-component" interventions were defined as those that combined two or more of the following strategies known as the "5 A's"

o Ask - Systematically identify all tobacco users at every visit

o Advise - Strongly urge all tobacco users to quit

o Assess - Determine willingness to make a quit attempt

o Assist - Aid the patient in quitting (provide counselling-style support and medication)

o Arrange - Ensure followup contact

Biochemical feedback

Various methods exist which allow a smoker to see the impact of their tobacco use, and the immediate effects of quitting ${ }^{12}$. Using biochemical feedback methods can allow tobacco-users to be identified and assessed and the use of monitoring throughout an effort to quit can increase motivation to quit.

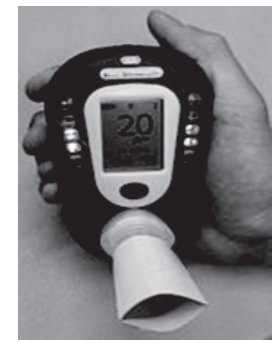

Breath CO monitor displaying carbon monoxide concentration of an exhaled breath sample (in ppm) with its corresponding percent concentration of carboxyhemoglobin.

- Breath carbon monoxide (CO) monitoring.

- Cotinine: A metabolite of nicotine, cotinine is present in smokers. Like carbon monoxide, a cotinine test can serve 
as a reliable biomarker to determine smoking status. Cotinine levels can be tested through urine, saliva, blood or hair samples.

Single medications

Single medications include:

Nicotine replacement therapy (NRT): Five medications approved by the U.S. Food and Drug Administration (FDA) deliver nicotine in a form that does not involve the risks of smoking.

1. Transderman Nicotine Patches.

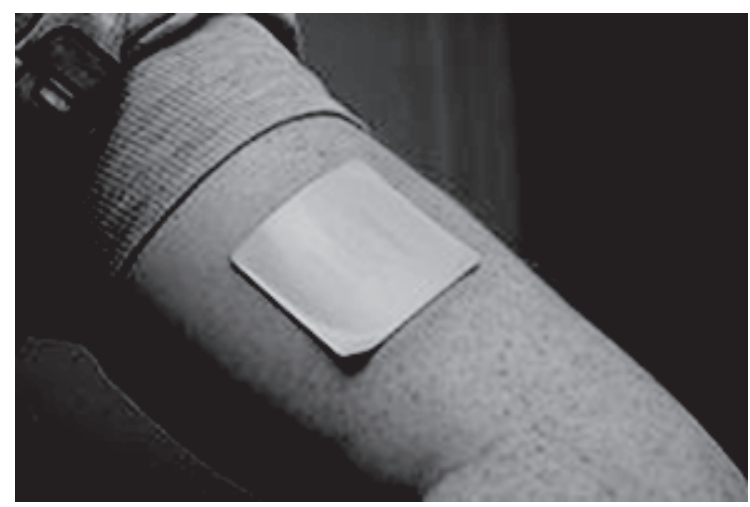

Nicoderm patch

All nicotine patches are placed and used in similar ways:

- A single patch is worn each day and replaced after 24 hours.

- Place the patch on different areas above the waist and below the neck each day.

- Put the patch on a hairless spot.

- People who wear the patches for 24 hours will have fewer withdrawal symptoms.

- If wearing the patch at night causes odd dreams, try sleeping without the patch.

- People who smoke fewer than 10 cigarettes per day should start with a lower dose patch (for example, $14 \mathrm{mg}$ ).

2. Gum

3. Lozenges

4. Spray

5. Inhaler

Antidepressant: Bupropion

Nicotinic receptorpartial agonists:

a) Cytisine is a plant extract that has been in use since the 1960s in former Soviet-bloc countries. It was the first medication approved as an aid to smoking cessation. b) Varenicline tartrate.

c) Moclobemide.

d) Two other medications have been used in trials for smoking cessation.

i. Clonidine may reduce withdrawal symptoms.

ii. Nortriptyline, another antidepressant, has similar success rates to bupropion. Combinations of medications

Three combinations of medications are effective

- Long-term nicotine patch and ad libitum NRT gum or spray

- Nicotine patch and nicotine inhaler

- Nicotine patch and bupropion (the only combination that the US FDA has approved for smoking cessation)

Other methods include:

Cut down to quit

Gradual reduction involves slowly reducing one's daily intake of nicotine. This can theoretically be accomplished through repeated changes to cigarettes with lower levels of nicotine, by gradually reducing the number of cigarettes smoked each day, or by smoking only a fraction of a cigarette on each occasion ${ }^{13}$.

Community interventions

Competitions and incentives

Psychosocial approaches

Self-help

Substitutes for cigarettes

- Electronic cigarette

- Chewing cinnamon sticks

- Plastic cigarette substitute

Alternative approaches

- Acupuncture

- Aromatherapy

- Hypnosis

- Herbs

\section{Discussion}

Nicotine is highly addictive.Nicotine addiction is the second-leading cause of death worldwide. The tar in cigarettes increases a smoker's risk of lung cancer, emphysema and bronchial disorders,atherosclerotic vascular disease. Pregnant smokers have a higher risk of miscarriage or low birth weight babies.Smoking also can contribute to other diseases, such as histiocytosis $\mathrm{X}$, obstructive sleep apnea, idiopathic pneumothorax and perinatal mortality. Cessation of smoking is not a easy 
method, it is associated with withdrawal symptoms. There are different methods of smoking cessation, like unassisted method, Single medication methods, combination methods, alternative methods etc. By applying these methods we can stop smoking and can get rid of a number of smoking related diseases and national costs.

\section{References}

1. Chapman S, MacKenzie $\mathrm{R}$. "The global research neglect of unassisted smoking cessation: causes and consequences".2010;24:205-7.

2. Benowitz NL; Benowitz, Neal L. "Nicotine addiction". N Engl J Med.2010;362 (24): 2295-303.

3. Cochrane Tobacco Addiction Group . 2011;13:15-7.

4. Fiore MC, Jaйn CR, Baker TB, et al . Clinical practice guideline: treating tobacco use and dependence.20011;12:561-4.

5. Fiore MC, Bailey WC, Cohen SJ, et al . Smoking cessation. Clinical practice guideline.2009;33:125-9.

6. Fiore MC, Bailey WC, Cohen SJ, et al . Clinical practice guideline: treating tobacco use and dependence.2011;65:23-9.
7. "Conflict over officials' stop-smoking advice".2008;45:56-8.

8. Ferguson J, Bauld L, Chesterman J, Judge K . "The English smoking treatment services: one-year outcomes".2008;2:59-69

9. American Cancer Society. 2011-01-31. Retrieved 2011-02-17.

10. Fiore MC, Novotny TE, Pierce JP, Giovino GA, Hatziandreu EJ, Newcomb PA, Surawicz TS, Davis RM . "Methods used to quit smoking in the United States. Do cessation programs help?". JAMA.2011;263 (20): 2760-5.

11. Baillie AJ, Mattick RP, Hall W . "Quitting smoking: estimation by meta-analysis of the rate of unaided smoking cessation". Aust J Public Health .2011;19 (2): $129-31$.

12. Lee CW, Kahende J. "Factors associated with successful smoking cessation in the United States, 2000". Am J Public Health.2007;97 (8): 1503-9.

13. Stead LF, Bergson G, Lancaster T, Stead, Lindsay F. ed. "Physician advice for smoking cessation". 2009;2:26-32. 